\section{The lateral tarsal strip in ectropion surgery: is it effective when performed in isolation?}

KYR Kam¹,2, CJ Cole', C Bunce ${ }^{3}$, MP Watson', D Kamal ${ }^{1}$ and JM Olver ${ }^{1}$

\section{Abstract}

Purpose The lateral tarsal strip (LTS) for involutional ectropion is often performed with a medial spindle (tarsoconjunctival diamond excision). We aimed to evaluate how well the LTS alone can achieve symptomatic relief. Methods A retrospective, comparative case series was performed on consecutive patients undergoing LTS alone or with medial spindle for involutional ectropion. Outcomes for LTS alone were clinically derived functional success (improvement in symptoms and aesthetic appearance) and anatomical success (judged by punctal position in the tear lake, punctal movement during blinking and absence of ectropion). We verified if these outcomes compared favourably with those of the patients who had undergone an LTS with medial spindle. Procedure selection was based on pre-operative clinical examination, especially the lateral pinch and twist test (this is described). Complications were also recorded. Results Of 67 eyes, 23 had LTS alone and 44 had LTS with a medial spindle. Of those having LTS alone the functional success rate was $87 \%(95 \%$ CI $(66.4,97.2 \%))$. This did not differ significantly from a success rate of $89 \%(75.4,96.2 \%)$ in LTS with a medial spindle $(P=0.99)$. A total of $78 \%(56.9,92.5 \%)$ of patients undergoing LTS had a good anatomical result compared with $82 \%(67.3,91.8 \%)$ of those who had an additional medial spindle $(P=0.75)$. Complication rates were similar between the groups.

Conclusion Where the lateral pinch and twist test returns the eyelid to a good position, the LTS alone can suffice for the management of involutional ectropion.

Eye (2012) 26, 827-832; doi:10.1038/eye.2012.34;

published online 9 March 2012
Keywords: involutional ectropion; lateral tarsal strip; medial spindle

\section{Introduction}

Lower lid ectropion is a very common eyelid pathology among the elderly and often results in troublesome symptoms and ocular surface complications. In involutional ectropion, the lower lid margin is everted off the surface of the globe as a result of structural eyelid changes that occur with increasing age, particularly laxity of the medial and lateral canthal tendons, disinsertion of the lower lid retractors, and orbicularis degeneration. ${ }^{1}$

A horizontal lower eyelid tightening procedure is often used when correcting ectropion. As lateral canthal tendon laxity is the primary problem in the majority of lax eyelids, the lateral tarsal strip (LTS), a procedure in which the lateral canthal tendon is tightened, is a well-recognised means for correcting paralytic or involutional upper and lower eyelid laxity, and lateral canthal tendon laxity or malposition. ${ }^{2}$ It is the preferred first-line operation in our unit for involutional ectropion with excess horizontal lid laxity. A medial spindle procedure is often performed in combination with the LTS in order to minimise any element of medial lid eversion and punctal malposition. Although there are clear theoretical benefits of adding a medial spindle, including the recruitment of the inferior canaliculus for tear drainage with surgical correction of punctal malposition, there has been no published literature confirming this theoretical advantage in practice or guiding the case selection for this adjunctive procedure. Moreover, our anecdotal experience suggests that patients in whom
${ }^{1}$ Oculoplastic and Orbital
Service, Western Eye
Hospital, London, UK

${ }^{2}$ Department of Surgery and Cancer, Imperial College London, London, UK

${ }^{3}$ Medical Statistics, Research and Development, Moorfields Eye Hospital NHS Foundation Trust, London, UK

Correspondence: JM Olver, Oculoplastic and Orbital Service, Western Eye Hospital, Marylebone Road, London NW1 5YE, UK Tel: + 44 (0)20 33123258 ; Fax: + 44 (0)20 33123259 . E-mail: janeolver@aol.com

Received: 22 July 2011 Accepted in revised form: 10 October 2011 Published online: 9 March 2012 
punctal positioning is not fully corrected still achieve adequate drainage of tears. An unpublished survey of ectropion management in the United Kingdom in 2004 performed by our department showed that oculoplastic surgeons perform the LTS alone more often than LTS with medial spindle.

The aim of our study was to analyze a cohort of patients who underwent lower lid surgery for involutional ectropion to determine what rates of symptomatic and anatomical improvement could be achieved in patients undergoing LTS alone.

We then sought to evaluate whether these success rates deviated substantially from those of patients who underwent LTS with medial spindle, and to assess complications occurring with the two procedures.

\section{Patients and methods}

The operating records of all patients on whom lower eyelid involutional ectropion surgery was performed between 1 May 1996 and 31 October 1999 were reviewed. Patients undergoing LTS with or without medial spindle were included. Patients with absent recording of baseline ectropion severity or post-operative functional or anatomic data were excluded. In patients with procedures performed bilaterally, we excluded the data from one eye on a random side to avoid bias. Patients who had undergone lower lid surgery in the past were included as long as they had not had a prior LTS: one patient had a wedge resection in the past and was included on this basis. All patients gave informed consent before surgery. Our local ethics committee confirmed that this study did not require ethical review.

Demographic data including age and sex were recorded, as were presenting symptoms described by the patient, and the baseline degree of severity of lower lid ectropion as graded in Table 1. Horizontal lid laxity was assessed by measuring the distractability of the lid forwards from the limbus using Castroviejo calipers and classified into groups of $>8 \mathrm{~mm}$ or $<8 \mathrm{~mm}$. Medial canthal tendon (MCT) laxity was tested by lateral distraction and graded 0-6 (Table 2). ${ }^{3}$ Any associated ocular disease including corneal, conjunctival, and nasolacrimal disease was also noted.

Table 1 Grading of severity of lower lid ectropion

\begin{tabular}{lc}
\hline Severity of ectropion & Number of patients at baseline (\%) \\
\hline Punctal eversion & $11(16.4 \%)$ \\
Medial $1 / 3-2 / 3$ ectropion & $33(49.3 \%)$ \\
$>2 / 3$ Ectropion & $16(23.9 \%)$ \\
Total tarsal eversion & $7(10.4 \%)$ \\
\hline
\end{tabular}

The grade of surgeon performing the operation was recorded as Specialist Registrar (SpR), Senior House Officer (SHO), Fellow or Consultant; in the UK, an $\mathrm{SHO}$ is a trainee in the first 2 years of specialist training (ST1-2), SpRs occupy the remaining years, and Fellows are subspecialty doctors in the year or two before Consultantship.

Table 2 Pre-operative features

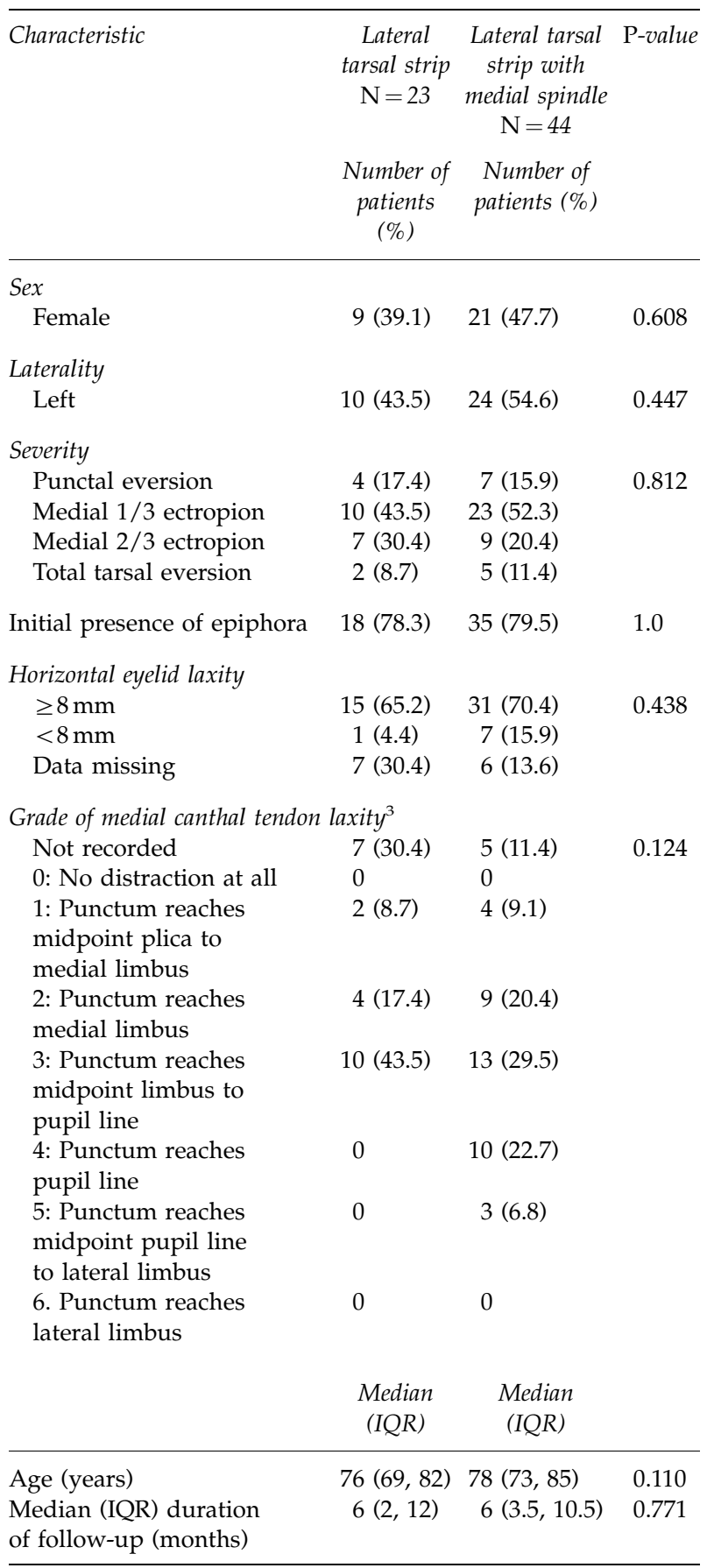

Abbreviation: IQR, interquartile range. 
The unit for years assumed that both a LTS and medial spindle were required for involutional ectropion (never medial spindle alone). Hence, all patients were listed routinely for LTS with a medial spindle. At surgery, the operating surgeon and/or the senior supervisor decided to omit the medial spindle if they felt this was unnecessary based on clinical re-examination, using a manoeuvre called the lateral pinch and twist test. The lateral lower lid was held by thumb and index finger, pulled laterally and inturned. If the lid and punctum clearly appeared anatomically apposed to the globe with this manoeuvre, it was decided that a LTS alone would suffice. This version of the lateral distraction test with additional inturning is used to predict the likely outcome of a LTS alone in ectropion patients, just like the thumb compression test in entropion patients. ${ }^{4}$ Thus, two groups of patients were created: those who had LTS alone, and those who had an additional medial spindle.

Our main outcome measures were post-operative anatomical and functional status, obtained and graded clinically at 6 weeks by members of the oculoplastic service, most often a surgeon who did not operate on the patient. Functional status incorporated aesthetic appearance of the lid with respect to erythema and inflammation, and reduction in patient symptoms (watering and irritation). Functional status was categorised as better, worse, or the same as pre-operative function. A good anatomic result was defined partly by punctal position in the tear lake, facing inwards or occasionally slightly upwards. The absence of ectropion or entropion and the absence of upward and outward movement of the punctum on blinking was also an indicator of good anatomic outcome. In contrast, the dynamic observation of the presence of this punctal movement on blinking, bringing the punctum medially out of the tear lake, would indicate a poor outcome.

Anatomic outcome was recorded as good, fair, or poor. We verified whether the outcomes in patients undergoing LTS alone compared favourably with those of patients who had LTS plus medial spindle, keeping in mind these were groups with differing pinch and twist test results at baseline.

Post-operative complications were compared between the groups and were classified as mild, moderate, or severe (defined in terms of the extent of morbidity involved and what sort of treatment was required as a consequence), and early (less than 6 weeks), intermediate (6 weeks to 6 months), or late (more than 6 months).

\section{Surgical technique of LTS}

We performed the technique outlined in reference 5 for the LTS.
A lateral canthal incision is made through skin and orbicularis. A lower limb lateral canthotomy and cantholysis is performed. The tarsal strip is fashioned and secured with 5:0 Ethibond to the zygomatic periosteum just inside the orbital rim at a level above the horizontal canthal line. The lateral canthal angle is reformed and the orbicularis and skin is closed in two layers.

\section{Surgical technique of medial spindle}

The details of this technique have been published by Nowinski and Anderson. ${ }^{6}$ The lower eyelid is everted and a lacrimal probe is inserted into the inferior canaliculus. A diamond shape of conjunctiva and lower eyelid retractors posterior to the lacrimal punctum is excised using Wescott scissors, so that the superior apex of the diamond is immediately below the lacrimal ampulla. The incision is closed with 6.0 Vicryl, during which the cut edge of the retractors is retracted to be included on the same needle pass as that which engages the conjunctiva at the lower apex of the diamond.

\section{Statistical analysis}

Baseline characteristics were compared between treatment groups using Fisher's Exact test for categorical variables and the Wilcoxon rank-sum test for continuous variables (because of marked departure from normality). Anatomical and functional outcomes are reported in the two operation groups and the proportion of patients achieving good results in the two operation groups is reported with $95 \%$ confidence intervals computed by the Exact Binomial method. A Fisher's exact test was used to assess for a difference in success rates between the operations. Logistic regression was used to assess whether there was any evidence of a difference in success rates after adjusting for baseline characteristics, that is, age, gender, and baseline severity of ectropion. To increase power, we conducted a sensitivity analysis - repeating our analyses but this time treating functional and anatomical outcomes as ordinal variables and using ordinal logistic regression (and found that results did not alter). All analyses were conducted using Stata 10.0 for Windows (Stata Statistical Software: Release 10, College Station, TX, USA).

\section{Statement of ethics}

We certify that all applicable institutional and governmental regulations concerning the ethical use of human volunteers or data were followed during this research. 
Table 3 Pre-operative symptoms and their distribution

\begin{tabular}{lc}
\hline Symptoms & $\begin{array}{c}\text { No. of patients (percentage of } \\
\text { study population) affected }\end{array}$ \\
\hline Horizontal eyelid sag & $54(81 \%)$ \\
Epiphora & $53(79 \%)$ \\
Discomfort & $10(15 \%)$ \\
Cosmetic problems & $11(16 \%)$ \\
Discharge/infections & $5(7 \%)$ \\
\hline
\end{tabular}

\section{Results}

A total of 91 patients were identified as having had lower eyelid involutional ectropion surgery. In all, 24 of these were excluded: 11 patients had missing notes, 9 had a Lazy $T$ operation or medial spindle alone, and 4 had incomplete notes. Statistical analysis was performed on the remaining 67 patients.

\section{Pre-operative features}

The LTS was performed in isolation on 23 patients $(34.3 \%)$, and 44 patients $(65.7 \%)$ had an additional medial spindle. Table 2 shows that baseline characteristics were not significantly different between the two groups. $80.6 \%$ (54) of all patients had data on horizontal eyelid laxity, 8 patients had eyelid laxity of $<8 \mathrm{~mm}$, and $46 \mathrm{had}>8 \mathrm{~mm}$ laxity. Of these 46,15 were in the LTS group. There was no difference in distribution between the two study groups with regards to MCT laxity $(P=0.124)$. Follow-up times were also similar. The distribution of baseline severity of ectropion is shown in Table 1: the most common presentation was medial $1 / 3$ to $2 / 3$ ectropion. Pre-operative symptoms and their prevalence are shown in Table 3.

A total of 30 operations (45\%) were performed by the $\mathrm{SpR}$, the consultant performed 17 operations (25\%), the fellow performed 14 operations (21\%), and the SHO performed 6 operations (9\%). There was no difference in the level of surgeon seniority between the two groups $(P=0.127)$.

\section{Analysis of outcomes}

Doing LTS alone produced good functional success rates (Table 4): post-operative function was better after $20 / 23$ ( $87 \%)$ of LTS operations $(95 \%$ CI $(66,97 \%)$. This symptomatic success rate did not deviate significantly from that of patients undergoing LTS plus medial spindle $(P=0.99): 39 / 44(89 \%)(95 \%$ CI $(75,96 \%))$. Logistic regression (binary and ordinal) showed no evidence of a difference in success rates after adjustment for baseline characteristics.
Table 4 Post-operative functional results

\begin{tabular}{|c|c|c|c|c|}
\hline \multicolumn{5}{|c|}{ Post-operative functional results } \\
\hline Operation & Better & Same & Worse & Total \\
\hline LTS & $20(87 \%)$ & $2(9 \%)$ & $1(4 \%)$ & 23 \\
\hline $\mathrm{LTS}+\mathrm{MS}$ & $39(89 \%)$ & $4(9 \%)$ & $1(2 \%)$ & 44 \\
\hline Total & $59(88 \%)$ & $6(9 \%)$ & $2(3 \%)$ & 67 \\
\hline \multicolumn{5}{|c|}{ Post-operative anatomic results } \\
\hline Operation & Good & Fair & Poor & Total \\
\hline LTS & $18(78 \%)$ & $3(13 \%)$ & $2(9 \%)$ & 23 \\
\hline $\mathrm{LTS}+\mathrm{MS}$ & $36(81.8 \%)$ & $6(13.6 \%)$ & $2(4.5 \%)$ & 44 \\
\hline Total & $54(81 \%)$ & $9(13 \%)$ & $4(6 \%)$ & 67 \\
\hline
\end{tabular}

Abbreviations: LTS, lateral tarsal strip; MS, medial spindle.

Anatomic results were also good in the majority of patients undergoing LTS alone (Table 4). We found little evidence of any difference in anatomic result compared to the patients who underwent LTS plus medial spindle $(P=0.753)$.

\section{Complications}

Table 5 shows the complications that occurred in both groups. Complication rates did not differ significantly between the two operations ( $P=0.501$ by Fisher's exact test). Although a significant proportion of patients suffered complications, most of these did not require further treatment. At 6 months the main problem was persistence of ectropion.

Punctal eversion recurred in three patients of the LTS group (two were asymptomatic) of whom one required a subsequent Lazy-T operation (this patient subsequently self-discharged). This compared to five patients in the group undergoing an additional medial spindle who were left with residual punctual eversion, none of whom were symptomatic enough to require further surgery. One of these patients had a wedge resection in the past. Four additional patients in the group who had a medial spindle had recurrence of ectropion, three of whom underwent redo operations (all successful).

In the LTS group, the patient who had wound dehiscence at the lateral canthal margin healed by secondary intention and was left with a persistent nontender granuloma, which needed no intervention. The patient who had in-turning eyelashes at the lateral canthus underwent a secondary procedure to open the lateral canthus and evert the lashes.

In the group of patients who had LTS combined with medial spindle, five patients had a small suture related granuloma that did not require surgery and two patients were left with a residual mild medial ectropion. 
Table 5 Complications of surgery

\begin{tabular}{|c|c|c|c|c|}
\hline & No complications & Mild complications & Moderate complications & Severe complications \\
\hline LTS & $12(52 \%)$ & $\begin{array}{l}4(17 \%) \\
\text { Conjunctivitis } \\
\text { Localised inflammation } \\
\text { Minor suture-related problems }\end{array}$ & $\begin{array}{l}7(30 \%) \\
\text { Recurrence of punctal eversion (3) } \\
\text { Periorbital haematoma } \\
\text { Wound dehiscence at lateral canthal margin } \\
\text { In-turning eyelashes at lateral canthus } \\
\text { Allergy to drops }\end{array}$ & $0(0 \%)$ \\
\hline LTS + MS & $15(34 \%)$ & $\begin{array}{l}11(25 \%) \\
\text { Bruising } \\
\text { Mild discharge } \\
\text { Grittiness } \\
\text { Bumpy feeling in lateral canthus } \\
\text { Blepharitis } \\
\text { Epiphora } \\
\text { Conjunctival chemosis (early) } \\
\text { Ingrowing lateral canthal lashes }\end{array}$ & $\begin{array}{l}16(36 \%) \\
\text { Recurrence of punctal eversion (9) } \\
\text { Visible ethibond suture } \\
\text { Small LTS dehiscence } \\
\text { Suture-related granuloma (5) }\end{array}$ & $\begin{array}{l}2(4.5 \%) \\
\text { Preseptal cellulitis } \\
\text { (two cases) }\end{array}$ \\
\hline
\end{tabular}

Abbreviations: LTS, lateral tarsal strip; MS, medial spindle.

Two patients had preseptal cellulitis and recovered uneventfully with a course of oral antibiotics.

\section{Discussion}

This is a unique investigation into whether the LTS alone is effective in treating involutional ectropion, presenting two large case series in parallel to verify the acceptability of outcomes compared to those achieved by LTS with medial spindle. Our results suggest that the LTS alone is effective in improving symptoms, cosmetic appearance and anatomy in patients who show good pre-operative lid and punctal apposition against the globe on performing a lateral pinch and twist test. Omitting a medial spindle in this group does not reduce the effectiveness of surgery, regardless of heterogeneity in other measures of ectropion severity. Patients whose lid position could not be adequately corrected in the lateral pinch and twist test underwent a LTS with medial spindle. Surgical outcomes in this latter group of patients showed that post-operative results in patients undergoing LTS alone were comparable.

An unresolved question is whether patients with an inadequate result with a lateral pinch and twist test may still respond to LTS alone. There is an assumption that the LTS should be done with a medial spindle but probably only a minority requires an additional medial spindle. It is probable that we have been overusing the medial spindle and that LTS alone, or other more modern canthoplasty procedure, is appropriate for the correction of the vast majority of cases of involutional ectropion. This issue will need to be tested in a future prospective and randomised trial.

We concede that due to the non-randomised and retrospective nature of our study there is a small possibility of selection bias. However, except for the result of the lateral pinch and twist test all other baseline features such as severity of ectropion were remarkably similar between our two groups of patients. In fact, all patients in this study were deemed suitable for LTS and were originally listed for LTS with a medial spindle. Although it cannot be concluded that performing LTS alone leads to the same result as performing LTS with a medial spindle, as we did not intend to compare these two groups directly, our study has demonstrated the efficacy of LTS as a standalone procedure and has shown that it produces comparable outcomes to a very similar group of patients undergoing LTS with medial spindle. While it is plausible that with larger numbers a difference in outcome between the groups might have been detected, the estimated odds ratio based on these data were close to unity (1.08 and 1.11 , respectively).

The aetiology underlying all ectropions in this study was involutional and not actinic, cicatricial or secondary to cosmetic aesthetic surgery such as blepharoplasty. We believe that a LTS alone suffices in most cases in improving functional success because the LTS restores the lower lid position against the globe, presenting the tear lake to the upper canaliculus during blinking and allowing adequate drainage to occur via this route even in the event of lower lid punctal malposition, which affected all patients at the outset. The LTS is a lateral canthoplasty that addresses both tarsal lengthening and lateral canthal tendon laxity.

Disadvantages of the LTS include lateral lash inturning, especially in older patients with anterior lamella slip - this occurred in two of our patients. Ache at the lateral canthus is often noticed for a few weeks postoperatively. There is also a small risk of suture related granuloma. Since this study we have changed our suture material to 5:0 Vicryl. Advantages of the LTS include repair of horizontal eyelid laxity by pulling the eyelid 
tightly against the globe, ${ }^{7}$ and the speed of the procedure. There is no danger of lid margin notching, minimal lid tissue is removed, phimosis is avoided, and canthal malposition and lid shortening are corrected simultaneously. ${ }^{8}$ Moreover, the almond shaped canthal angle is preserved by substituting a strip of tarsus for the lateral canthal tendon, ${ }^{9}$ there is a good cosmetic result and post-operative rehabilitation is rapid. Results were sustained long-term in the majority of the patients in our study, that is, recurrence of canthal tendon laxity and elongation that may occur more frequently following traditional shortening procedures seems to be less common after LTS.

The addition of a medial spindle procedure is not without potential disadvantages. It can pull the medial lower lid margin contour downwards and can also lead to irritation, redness, conjunctival inflammation and mucus production. If it could be safely omitted, an extra step could be avoided and the ectropion repair could be kept as simple as possible.

We found that four $(6 \%)$ of our patients had associated nasolacrimal duct pathology, which shows the importance of assessing the lacrimal system thoroughly before operation. A patient who complains of epiphora and has nasolacrimal blockage would derive little benefit from lower eyelid surgery unless the nasolacrimal pathology was addressed first.

In conclusion, this study has determined that the LTS alone is effective in a large subset of patients undergoing surgery for involutional ectropion. A safe method of determining if patients are suitable for omission of further procedures such as a medial spindle would be to check if good lid position is attainable using the lateral pinch and twist test. We hope that this study will promote larger scale, randomised, prospective studies to verify our findings and further define procedure selection for involutional ectropion surgery.

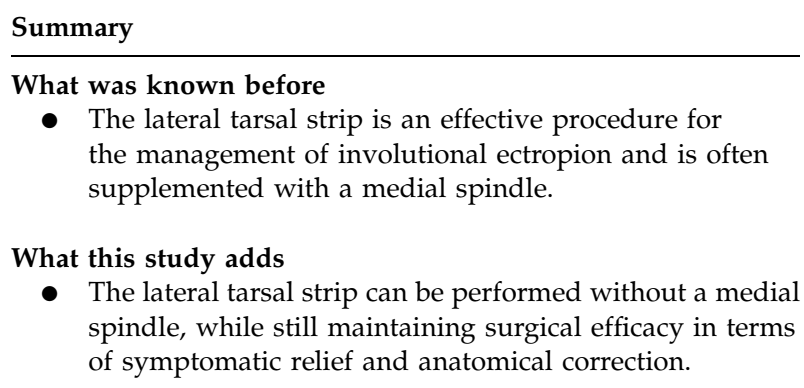

\section{Conflict of interest}

The authors declare no conflict of interest.

\section{References}

1 Vallabhanath P, Carter SR. Ectropion and entropion. Curr Opin Ophthalmol 2000; 11: 345-351.

2 Weber PJ, Popp JC, Wulc AE. Refinements of the tarsal strip procedure. Ophthalmic Surg 1991; 22: 687-691.

3 Olver JM, Sathia PJ, Wright M. Lower eyelid medial canthal tendon laxity grading: an interobserver study of normal subjects. Ophthalmology 2001; 108: 2321-2325.

4 Olver JM, Barnes JA. Effective small-incision surgery for involutional lower eyelid entropion. Ophthalmology 2000; 107: 1982-1928.

5 Olver JM. Surgical tips on the lateral tarsal strip. Eye 1998; 12: 1007-1012.

6 Nowinski TS, Anderson RL. The medial spindle procedure for involutional medial ectropion. Arch Ophthalmol 1985; 103: 1750-1753.

7 Dryden RM, Edelstein JP. Lateral palpebral tendon repair for lower eyelid ectropion. Ophthalmic Plast Reconstr Surg 1988; 4: 115-118.

8 Jordan DR, Anderson RL. The lateral tarsal strip revisited. The enhanced tarsal strip. Arch Ophthalmol 1989; 107: 604-606.

9 Anderson RL, Gordy DD. The tarsal strip procedure. Arch Ophthalmol 1979; 97: 2192-2196. 